\title{
ILUSTRAÇÃO DA INFLUÊNCIA DA RAZÃO DE AQUECIMENTO NOS RESULTADOS DE TERMOGRAVIMETRIA
}

\section{Jivaldo do Rosário Matos, Miriam Hisami Miyano e Lilian Siqueira}

Instituto de Química - Universidade de São Paulo - Av. Prof. Lineu Prestes, 748 - 05508-900 - São Paulo - SP

Maria de Fátima Vitória de Moura

Departamento de Química - Centro de Ciências Exatas e da Terra - UFRN - CP 1662 - 59072-970 - Natal - RN

José Marques Luiz

Faculdade de Engenharia de Guaratinguetá - UNESP - CP 205 - 12500-000 - Guaratinguetá - SP

Recebido em 20/10/98; aceito em 11/5/99

\begin{abstract}
ILUSTRATION OF THE INFLUENCE OF THE HEATING RATE IN THERMOGRAVIMETRY RESULTS. The effects of the heating rate on a TG curve of a sample have been widely discussed in the literature. This paper shows the influence of heating rate $(\beta)$ in thermogravimetry results when stoichiometry determinations are studied. For this purpose the compound $\operatorname{Sm}\left(\mathrm{CH}_{3} \mathrm{SO}_{3}\right)_{3} .2(3-\mathrm{picNO})$ was considered $\beta$ of 2,$5 ; 5 ; 10 ; 20$ and $40^{\circ} \mathrm{C} \mathrm{m^{-1 }}$ in dynamic air atmosphere were obtained. The results are in agreement with proposed stoichiometry for $\beta$ equal to 2,5 and $5^{\circ} \mathrm{C} \mathrm{min}^{-1}$. However, using a higher $\beta$ the same results were not obtained resulting in false stoichiometry determinations.
\end{abstract}

Keywords: heating rate; thermogravimetry; stoichiometry determination.

\section{INTRODUÇÃO}

Como em qualquer técnica instrumental, na análise térmica são inúmeros os fatores que afetam a natureza, precisão e exatidão dos resultados experimentais.

Dentre as técnicas de análise térmica, a termogravimetria (TG) provavelmente é a que apresenta maior número de variáveis, devido à natureza dinâmica da variação de massa da amostra. Vários estudos têm discutido em detalhes as precauções e cuidados que devem ser tomados na utilização da termobalança $\mathrm{a}^{1-3}$, bem como de muitas outras variáveis envolvidas na termogravimetria. Basicamente, os parâmetros que influenciam esses resultados são atribuídos a fatores instrumentais e relacionados com as características da amostra.

Os fatores instrumentais (da termobalança) são devidos a razão de aquecimento, atmosfera do forno, composição do porta-amostra e a geometria do porta-amostra e do forno, enquanto as características da amostra são devidas à quantidade de amostra, solubilidade dos gases libertados na amostra, natureza da amostra e condutividade térmica.

Dos fatores enumerados acima, o efeito da razão de aquecimento $(\beta)$ nas curvas TG é o mais amplamente estudado. Geralmente, à medida que aumenta $\beta$ há um deslocamento da temperatura de termodecomposição para valores mais elevados ${ }^{4}$. A detecção de compostos intermediários a partir das curvas TG também depende da $\beta$ utilizada, bem como da natureza da amostra.

O objetivo desse trabalho é alertar quanto a interpretação inadequada das etapas de decomposição térmica por termogravimetria, ao considerar os dados obtidos utilizando apenas uma razão de aquecimento e, ainda, buscar uma correlação com os dados de calorimetria exploratória diferencial (DSC)

\section{PARTE EXPERIMENTAL}

Os metanossulfonatos de terras raras hidratados, $\mathrm{Ln}\left(\mathrm{CH}_{3} \mathrm{SO}_{3}\right)_{3}$. $\mathrm{xH}_{2} \mathrm{O}$, reagem com o ligante 3-picolina-N-óxido (3-picNO) formando compostos isomorfos de composição $\mathrm{Ln}\left(\mathrm{CH}_{3} \mathrm{SO}_{3}\right)_{3} 2$ (3picNO), onde $\mathrm{Ln}$ representa $\mathrm{La}-\mathrm{Yb}$ e $\mathrm{Y}$ (exceto $\mathrm{Pm}$ ). A preparação desses compostos encontra-se descrita na literatura ${ }^{5,6}$.

Para o presente estudo foi sintetizado o composto $\mathrm{Sm}\left(\mathrm{CH}_{3} \mathrm{SO}_{3}\right)_{3}$. 2(3-picNO). A determinação do teor de metal foi feita a partir da complexometria com EDTA, conforme técnica descrita por Lyle e
$\operatorname{Rahman}^{7}$ que consideram a faixa de $\mathrm{pH} 5,8$ a 6,4 como a mais satisfatória e conveniente para a titulação direta, com algumas modificações propostas por Osório e Feitosa ${ }^{8}$ em relação a quantidade de solução tampão ácido acético / acetato de sódio.

Os teores de $\mathrm{C}, \mathrm{H}$ e $\mathrm{N}$ foram determinados a partir do equipamento Elemental Analyser CHN, modelo 2400 marca Perkin Elmer.

A medida do intervalo de fusão do composto foi obtido no aparelho Cappillary Melting Point Apparatus marca Thomas Hoover.

As curvas TG/DTG foram obtidas na termobalança TGA-50 marca Shimadzu. Nos ensaios utilizou-se massa de amostra da ordem de $4 \mathrm{mg}$, cadinho de platina, atmosfera dinâmica de ar $(50 \mathrm{~mL} / \mathrm{min})$ e razões de aquecimento $(\beta)$ de 2,$5 ; 5 ; 10 ; 20$ e $40^{\circ} \mathrm{C} / \mathrm{min}$, no intervalo de temperatura de 25 a $600^{\circ} \mathrm{C}$. A análise das curvas TG/DTG foi feita descontando-se uma curva em branco, obtida nas mesmas condições experimentais das amostras, porém com o cadinho vazio.

As curvas DSC foram obtidas a partir da célula DSC-50 (Mettler TA 4000 System). Nos ensaios, utilizou-se massa de amostra da ordem de $2 \mathrm{mg}$, cápsula de alumínio, atmosfera de ar (vazão de $50 \mathrm{~mL} / \mathrm{min}$ ) e $\beta$ de 2,$5 ; 5 ; 10 ; 20$ e $40^{\circ} \mathrm{C} / \mathrm{min}$. O equipamento foi previamente calibrado utilizando como padrões os metais índio e zinco, ambos de pureza 99,99\%. A partir das medidas de ponto de fusão desses metais, índio $\left(156,6^{\circ} \mathrm{C}\right)$ e zinco $\left(419,6^{\circ} \mathrm{C}\right)$ foi feita a calibração de temperatura. O fluxo de calor, $\Delta \mathrm{H}$, foi ajustado com base no calor de fusão do índio metálico que é de $28,59 \mathrm{~J} / \mathrm{g}$. Foram calculados os fatores de correção conforme procedimento e especificação do fabricante da Mettler. Os valores de $\Delta H$ das amostras foram obtidos a partir da área dos picos das curvas DSC.

\section{RESULTADOS E DISCUSSÃO}

O estudo do comportamento térmico de vários compostos formados pelo grupo metanossulfonato $\left(\mathrm{MS}^{-}\right)$com metais de transição $^{9-15}$, terras raras ${ }^{16,17}$, e composições mais complexas envolvendo além da espécie $\mathrm{Ln}\left(\mathrm{CH}_{3} \mathrm{SO}_{3}\right)_{3}$, ligantes adicionais coordenados aos íons de terra raras ${ }^{18}$ tem sido descrito na literatura.

Estudos relativos à síntese, caracterização e ao comportamento térmico de complexos obtidos entre metanossulfonatos de terras raras com os ligantes 3-picNO foram previamente 
Tabela 1. Teores de $\mathrm{Sm}, \mathrm{C}, \mathrm{H}$ e $\mathrm{N}$ e faixa de fusão aparente do compostoSm $\left(\mathrm{CH}_{3} \mathrm{SO}_{3}\right)_{3}$. 2(3-picNO).

\begin{tabular}{|c|c|c|c|c|c|c|c|c|}
\hline \multicolumn{2}{|c|}{$\% \mathrm{Sm}$} & \multicolumn{2}{|c|}{$\% \mathrm{C}$} & \multicolumn{2}{|c|}{$\% \mathrm{H}$} & \multicolumn{2}{|c|}{$\% \mathrm{~N}$} & \multirow{2}{*}{$\begin{array}{c}\mathrm{T}_{\text {fusão aparente }} \\
\left({ }^{\circ} \mathrm{C}\right)\end{array}$} \\
\hline Calc. & Exp. & Calc. & Exp. & Calc. & Exp. & Calc. & Exp. & \\
\hline 22,29 & 22,23 & 27,55 & 27,13 & 3,54 & 3,28 & 4,28 & 4,24 & $212-218$ \\
\hline
\end{tabular}

abordados ${ }^{19}$. Nesses estudos, observaram-se que tanto os ânions $\mathrm{MS}^{-}$como os ligantes 3-picNO estão coordenados ao íon lantanídeo. O comportamento térmico desses compostos foi investigado em atmosferas de ar e nitrogênio, mostrando que a formação da espécie $\mathrm{Ln}\left(\mathrm{CH}_{3} \mathrm{SO}_{3}\right)_{3}$ ocorre, em temperaturas entre 250 e $300^{\circ} \mathrm{C}$, após a saída das duas moléculas do ligante 3-picNO.

Para o composto objeto deste estudo, a Tabela 1 mostra que os resultados experimentais obtidos na determinação do teor de Sm, C, H e N apresentaram-se concordantes com os valores teóricos calculados, confirmando a estequiometria proposta. Nessa tabela, também, está listado o intervalo de fusão aparente do composto.

A Figura 1, apresenta as curvas TG do composto $\mathrm{Sm}\left(\mathrm{CH}_{3} \mathrm{SO}_{3}\right)_{3} .2$ (3-picNO) obtidas sob diferentes razões de aquecimento $(\beta)$. Observa-se que na menor $\beta$ o processo de libertação das moléculas do ligante, 3-picNO, ocorre em uma única etapa (Figura 2), e os cálculos de variação de massa $(\Delta \mathrm{m})$ experimental $(33,25 \%)$ e calculado $(33,38 \%)$ apresentaram-se concordantes (Tabela 2).

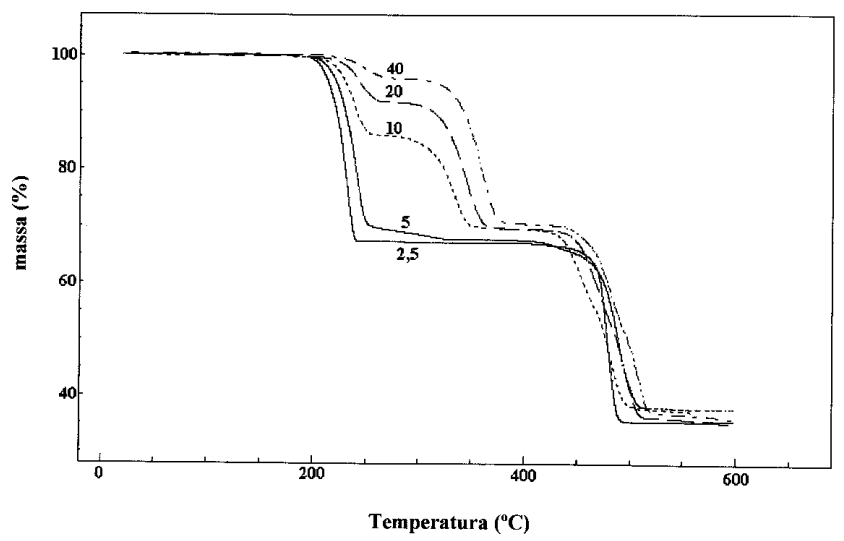

Figura 1. Curvas $\mathrm{TG}$ do $\mathrm{Sm}\left(\mathrm{CH}_{3} \mathrm{SO}_{3}\right)_{3} .2(3-p i c \mathrm{NO})$ obtidas em atmosfera de ar $(50 \mathrm{ml} \mathrm{min}-1) ; \beta=2,5 ; 5 ; 10 ; 20 ; 40^{\circ} \mathrm{C} \mathrm{min}^{-1}$.

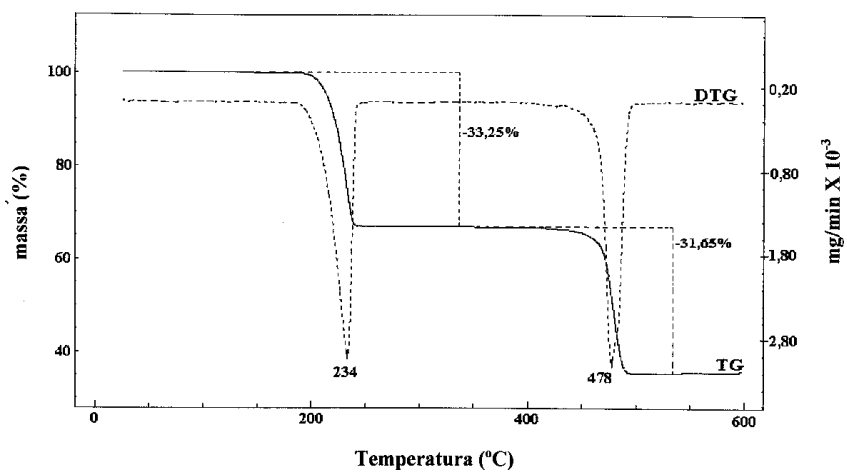

Figura 2. Curvas TG/DTG do $\mathrm{Sm}\left(\mathrm{CH}_{3} \mathrm{SO}_{3}\right)_{3}$.2(3-picNO) obtidas em atmosfera de ar (50ml min); $\beta=2,5^{\circ} \mathrm{C} \mathrm{min}^{-1}$.

No entanto, para $\beta$ maiores (Figura 1 e 3 ) esse processo ocorre em duas etapas, de modo que a fração de ligante libertada, entre as etapas, diminui com o aumento de $\beta$ e o somatório dos valores de $\Delta \mathrm{m}$ se afasta do valor teórico calculado de $33,38 \%$ (Tabela 2), ocorrendo uma diminuição em função do processo de carbonização parcial do ligante. $\mathrm{O}$ intervalo de temperatura correspondente
Tabela 2. Valores de variação de massa $(\Delta \mathrm{m})$ no intervalo de temperatura $(\Delta \mathrm{T})$ correspondente a libertação do aminóxido aromático no composto $\mathrm{Sm}\left(\mathrm{CH}_{3} \mathrm{SO}_{3}\right)_{3}$. 2(3-picNO) em diferentes razões de aquecimento $(\beta)$.

\begin{tabular}{ccc}
\hline$\beta\left({ }^{\circ} \mathrm{C} / \mathrm{min}\right)$ & $\Delta \mathrm{T}\left({ }^{\circ} \mathrm{C}\right)$ & $\Delta \mathrm{m}(\%)$ \\
\hline 2,5 & $130-340$ & 33,25 \\
5 & $140-370$ & 32,53 \\
10 & $150-400$ & 30,60 \\
20 & $160-400$ & 30,53 \\
40 & $170-415$ & 30,02 \\
\hline
\end{tabular}

à libertação do aminóxido aromático varia de forma que, os extremos dessa faixa, temperatura inicial e final, são deslocados para temperaturas maiores com o aumento de $\beta$. Com isso, atinge-se, rapidamente, uma alta temperatura sem que haja tempo suficiente para libertação total do ligante, e assim, as moléculas remanescentes sofrem este processo da carbonização. Observou-se, também, uma diminuição na faixa de estabilidade térmica da espécie $\mathrm{Sm}\left(\mathrm{CH}_{3} \mathrm{SO}_{3}\right)_{3}$ com o aumento de $\beta$.

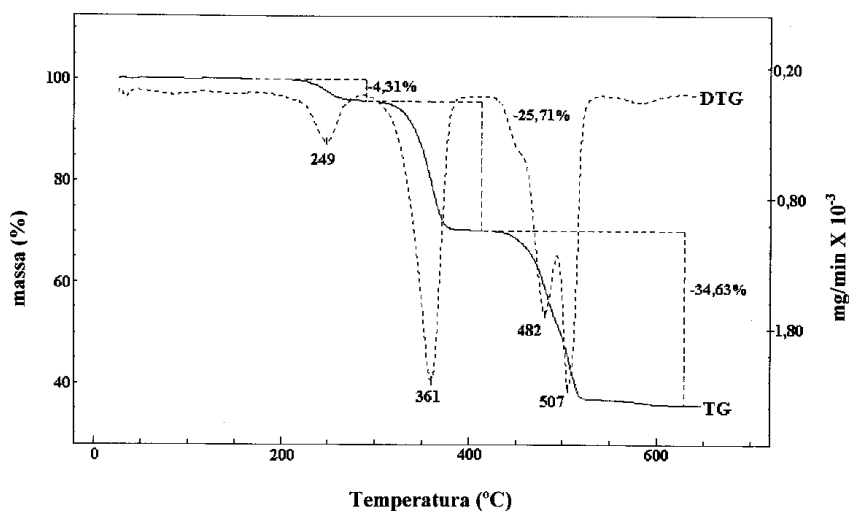

Figura 3. Curvas TG/DTG do $\mathrm{Sm}\left(\mathrm{CH}_{3} \mathrm{SO}_{3}\right)_{3}$.2(3-picNO) obtidas em atmosfera de ar $(50 \mathrm{ml} \mathrm{min}-1) ; \beta=40^{\circ} \mathrm{C} \mathrm{min}^{-1}$.

As curvas DSC (Figura 4) obtidas com diferentes $\beta$ mostram a presença de um pico correspondente a um evento endotérmico, relacionado com a fusão e decomposição térmica do composto. Uma vez que na temperatura do pico da curva DSC, as curvas TG/DTG evidenciam perda de massa. A Tabela 3 , apresenta os valores da temperatura do pico e o valor de $\Delta \mathrm{H}$ correspondente a esse evento. Observa-se que, de maneira geral, há um aumento dos valores de temperatura correspondentes a esses eventos, e uma diminuição dos valores de $\Delta \mathrm{H}$, com o aumento de $\beta$. Isto ocorre porque aumentando $\beta$, parte do ligante carboniza antes da volatilização, e isso não envolve gasto de calor para libertá-lo. Essa diminuição nos valores de $\Delta \mathrm{H}$ está em concordância com a variação ocorrida com os percentuais de perda de massa observada nas curvas TG/DTG.

\section{CONCLUSÕES}

Alguns fatores devem ser considerados na utilização das técnicas termoanalíticas. Na termogravimetria, a razão de aquecimento pode deslocar os eventos, para temperaturas maiores ou menores, bem como influenciar no número de etapas de decomposição térmica, causar variações nos valores de perda 
Tabela 3. Valores de temperatura do pico endotérmico de fusão com decomposição térmica e valores de $\Delta \mathrm{H}$ obtidos a partir das curvas DSC em diferentes razões de aquecimento $(\beta)$.

\begin{tabular}{ccc}
\hline$\beta\left({ }^{\circ} \mathrm{C} / \mathrm{min}\right)$ & $\mathrm{T}_{\text {pico }}\left({ }^{\circ} \mathrm{C}\right)$ & $\Delta \mathrm{H}(\mathrm{kJ} / \mathrm{mol})$ \\
\hline 2,5 & 207,8 & 232,4 \\
5 & 216,7 & 230,8 \\
10 & 225,1 & 227,1 \\
20 & 226,9 & 178,5 \\
40 & 225,6 & 132,0 \\
\hline
\end{tabular}

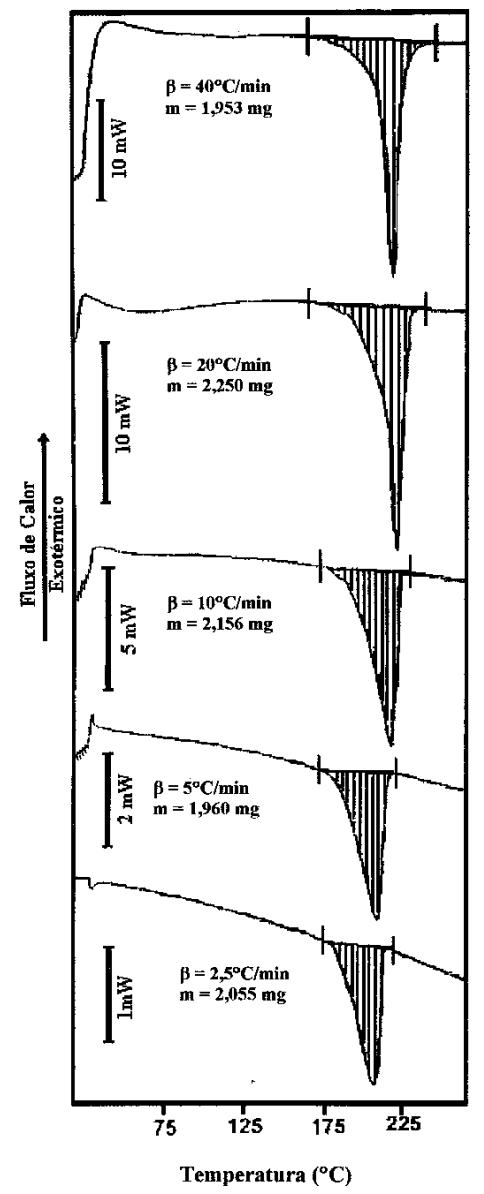

Figura 4. Curvas DSC sob atmosfera dinâmica de ar e diferentes razões de aquecimento do composto $\mathrm{Sm}\left(\mathrm{CH}_{3}\right)_{3}$.2(3-picNO). de massa, induzindo a erros quando se pretende definir a estequiometria de uma dada espécie. Similarmente, ocorre com a calorimetria exploratória diferencial que, ainda, pode sofrer variações nos valores de $\Delta \mathrm{H}$, corroborando com os dados da termogravimetria. Dessa forma, no estudo do comportamento térmico de uma determinada espécie, é aconselhável que sejam investigadas diferentes razões de aquecimento.

\section{AGRADECIMENTOS}

Os autores agradecem ao CNPq e FAPESP pelos auxílios recebidos, à Sinc do Brasil pelo suporte técnico, e ao Instituto de Química da USP-São Paulo.

\section{REFERÊNCIAS}

1. Duval, C.; Inorganic Thermogravimetric Analysis; Elsevier; $2^{\text {nd }}$ ed.; Amsterdam, 1963.

2. Wendlandt, W.W.; Thermal Analysis; Wiley; $3^{\text {rd }}$ ed.; New York, 1985.

3. Haines, P. J.; Thermal Methods of Analysis - Principles, Aplications and Problems; Champman and Hall; $1^{\text {st }}$ ed; London, 1995.

4. Simons, E. L.; Newkirk, A. E.; Talanta 1964, 11, 549.

5. Matos, J. R.; Dissertação de Mestrado, Instituto de Química, USP, São Paulo, 1984.

6. Zinner, L. B. and Matos, J. R.; Anais do VIII Simpósio Anual da ACIESP, São Paulo 1984, 1, 104.

7. Lyle, S. J.; Rahman, M. D.; Talanta 1963, 10, 1177.

8. Osório, V. K. L.; Feitosa, M. L.; Anais do VI Simpósio Anual da ACIESP, São Paulo 1982, 1, 277.

9. Charbonnier, F.; Ann. Chim. 1971, 6, 405.

10. Charbonnier, F.; Gauthier, J.; C. R. Seances Acad. Sci. 1969, 268, 1596.

11. Charbonnier, F.; Gauthier, J.; C. R. Seances Acad. Sci. 1970, 271, 830.

12. Charbonnier, F.; Thermochim. Acta. 1973, 7, 217.

13. Charbonnier, F.; J. Therm. Anal. 1974, 6, 45.

14. Charbonnier, F.; Thermochim. Acta. 1979, 33, 31.

15. Charbonnier, F.; Faure, F.; Loiseleur, H.; H. Rev. Chim. Miner. 1979, 16, 335.

16. Zinner, L. B.; An. Assoc. Bras. Quim. 1979, 30, 27.

17. Zinner, L. B.; An. Assoc. Bras. Quim. 1980, 52, 715.

18. Zinner, L. B.; Vicentini, G.; J. Inorg. Nucl. Chem. 1980, 42, 1349.

19. De Matos, J. E. X.; Niinistro, L.; Matos, J. R.; Vicentini, G.; Zinner, L. B.; Acta Chem. Scand. 1988, A 42, 111. 